\title{
Les déplacements de la menace: (fé-)néantisation du nord par le nord
}

Denis Duclos

\section{(2) OpenEdition \\ 1 Journals}

Édition électronique

URL : http://journals.openedition.org/conflits/103

DOI : $10.4000 /$ conflits. 103

ISSN : $1777-5345$

Éditeur :

CCLS - Centre d'études sur les conflits lilberté et sécurité, L'Harmattan

Édition imprimée

Date de publication : 17 mai 1991

ISSN : 1157-996X

Référence électronique

Denis Duclos, «Les déplacements de la menace: (fé-)néantisation du nord par le nord 》, Cultures \& Conflits [En ligne], 02 I printemps 1991, mis en ligne le 30 décembre 2002, consulté le 30 mars 2021. URL : http://journals.openedition.org/conflits/103 ; DOI : https://doi.org/10.4000/conflits.103

Ce document a été généré automatiquement le 30 mars 2021.

Creative Commons License 


\title{
Les déplacements de la menace: (fé-)néantisation du nord par le nord
}

\author{
Denis Duclos
}

1 "Tout schème, si fondamental qu'il puisse paraître, engendre normalement son contraire, que ce soit par l'effet immédiat d'un jeu de miroir, ou par celui d'une élaboration à terme". (C. Lévi-Strauss, L'Homme nu, p. 545.)

Du "Pont" Est-Ouest au "Puits" Nord-Sud : les avatars de la métaphore

2 Pour la cosmologie symbolique, qui n'a qu'un rapport lointain est-il besoin de le dire, avec l'astronomie scientifique, le globe terrestre est assis dans l'espace, en équilibre sur le pôle Sud. Il devient un corps, certes sphérique, mais qui est imaginé avec les potentiels de latéralisation et de verticalisation d'un organisme humain. Malgré nous, et tout en demeurant capables d'une géographie rationnelle, nous laissons souvent agir d'elles-mêmes d'étranges indexations de la réalité politique ou sociale, par un cosmos au moins aussi fantastique que celui des Anciens(1).

Il est à remarquer que cette métaphorisation est le support de deux opérations : une extrême simplification(2) et une expression de l'antagonisme. Dans la version la plus simple, ce que je n'hésiterai pas à appeler le délire de l'hypothèse "Gaïa", la surface vivante du globe peut devenir réfractaire à l'anthroposphère - sorte de pellicule irritante d'activités agitées et polluantes - et se débarrasser de l'humain pour préserver ses équilibres. Dans les modèles dits "géopolitiques", l'unité planétaire, bien que de mieux en mieux entrevue depuis l'oeil distancié des satellites militaires, reste pour ainsi dire dérobée à l'imagination par le phénomène de la polarisation organique. Les quatre points cardinaux ne sont pas ce que le petit bonhomme des manuels scolaires symbolise en regardant le Nord pour situer Est et Ouest de ses bras étendus, tout en marchant sur le Sud : c'est le contraire qui se produit. Le Nord devient tête, le Sud, pieds, et l'Est-Ouest, la dimension où se déploie l'axe de symétrie du buste et des membres. 
Comme surface d'antagonismes Est-Ouest, c'est-à-dire latéralisés, la planète est donc presque automatiquement perçue comme lieu d'un redoublement d'organismes identiques glissant côte à côte. Les variations cartographiques les plus ingénieuses comme celles proposées par Chaliand) ne changent rien à cette propension imaginaire à considérer l'ancien et le nouveau monde, les deux masses continentales géantes de l'hémisphère Nord, notamment, comme des êtres situés en regard les uns des autres, au même niveau, à la fois partagés et cernés par leur propre manière de ceinturer la planète. Comme si l'histoire de la dérive des continents devait elle-même dériver pour appuyer le fantasme d'un apparentement égalitaire entre Est et Ouest, simple forme de la symétrie Gauche- Droite. C'est pourquoi, lorsque la menace change de polarité, et d'Est-Ouest devient Nord-Sud, il se produit un changement inconscient dans sa structure. M. Serres dirait que nous passons du Pont au Puits(3).

5 Nous ne sommes plus en effet dans le registre des frères ennemis, de la duplication des semblables. Nous entrons dans celui de la division interne d'un organisme ou un système, dans sa découpe "sagittale", entre un haut, un milieu et un bas'.

De métaphorique, l'activité symbolique devient métonymisante(5): elle traite le rapport Nord-Sud comme un système d'emboittements d'enfoncements, de partitions de soi-même. Si l'on osait (et il semble bien que ce soit pratiquement le cas), les parties hautes (Nord) sentent que "leur" Sud leur correspondent en propre et de façon spécifique, comme des jambes correspondent au reste de l'organisme qui s'y appuie. Le conflit Est-Ouest était une histoire de miroir, d'amour-haine, dans laquelle la question était de savoir si nous étions menacés par notre propre image décalée, par d'autres Occidentaux, des frères légèrement déplacés vers l'Est. La guerre Nord-Sud devient au contraire une affaire de surplomb, de haut en bas, et réciproquement.

7 Or, dans notre société moderne privilégiant la tête comme le lieu même de l'humain, le haut glisse vite vers ce qu'on désigne par le plus important, le noble, l'englobant, le pensant, et le bas, vers ce qui est trivial, mais aussi dépendant, aliéné à ce qui le dirige à l'intérieur du même Oikos. On y retrouve avec familiarité les paraboles du Maître et de l'Esclave, et avec elles le phantasme hégélien très classique du "servant" qui, par les voies d'un travail acharné sur lequel repose le confort du maître, finit par remplacer celui-ci, trop purement guerrier.

Bien entendu, ceux qui se rallient à la métonymie Nord-Sud peuvent affirmer qu'ils n'y mettent aucune malignité, et qu'au contraire cette gestalt simplifiante peut aider à mettre sur pied un système de relations plus franc et plus global, donc plus cohérent. .le crois que c'est faux, quelle que soit l'ambition qui a pu présider au choix de cet étiquetage" : car nous ne maîtrisons pas aisément les effets de langage et d'image. Ce sont plutôt eux qui nous maîtrisent, et ce n'est pas un hasard si, avec l'adoption de la métonymie Nord-Sud, les concepts plus articulés de développement, de tiers ou de quart monde, de régions, de non-alignement de nouvel ordre économique mondial, etc., se trouvent sinon désactivés, du moins en partie désamorcées(6).

9 Comme l'avait déjà observé K. Mannheim à la veille de la Seconde Guerre mondiale, l'introduction d'un symbole simplificateur s'opère toujours d'abord de façon innocente, comme s'il s'agissait dune pure substitution pratique à d'autres concepts moins opératoires(7). Puis ce symbole "devient la nouvelle force conductrice pour des formes nouvelles d'intégration spontanée des groupes (c'est la période utopienne du symbole). Enfin le symbole devient l'emblème rigide d'un groupe organisé". On assiste du même coup, écrit Mannheim, à un passage "de l'insécurité inorganisée à l'insécurité 
organisée" par cette transformation du système symbolique en trois étapes, qui endentifie, fixe au support symbolique arbitrairement choisi l'identité du groupe, désormais tenue pour "évidente". Je crois que l'opposition symbolique Nord-Sud a subi une transformation de ce type, depuis le milieu des années soixante-dix lorsque dans des milieux intellectuels contestant le tiers-mondisme classique aux Etats-Unis, des auteurs comme R. D. Hansen(8) ont commencé à marteler cette notion, qui avait déjà la faveur de la Commission Trilatérale(9).

Notons bien que ce glissement sémantique se proposa d'abord comme le moyen d'un meilleur réalisme négociatif, pour une meilleure équité entre riches et pauvres, et pour envisager de satisfaire au mieux "les besoins humains de base", etc. Mais, rapidement, la disponibilité de ce dualisme à la confrontation pointa le bout de l'oreille. Ainsi, dans un ouvrage significatif de l'époque (par ses bonnes intentions caritatives rationalisées(10)), on observe que les locutions utilisant le couple Nord-Sud dérivent au fil des pages vers la confrontation de deux éthos incompatibles : ce qu'on appelle déjà "Les puissances du Nord et du Sud" divergent de façon reconnaissable : au Nord, des pays "responsables" "observent avec inquiétude" des "régimes du Sud déplaisants" (p. 155); il y a une "lutte Nord-Sud pour un meilleur partage" (157), mais ce sont des "consciences" qui au Nord s'opposent aux "pauvres" du Sud. Certes, la notion de menace était certes déjà fort active(11) avant que d'être rapportée à l'antagonisme Nord-Sud: mais appliquée au Tiers-Monde, elle attirait encore l'esprit sur la crainte matérielle des dégâts liés à la pauvreté ; réarticulée sur l'opposition Nord-Sud, elle devenait en revanche plus ambiguë, plus globale, plus disponible à la polarisation du registre militaire.

Les contradictíons du mythe Nord-Sud

11 Mais l'accomplissement de la dialectique classique nous confronte à un paradoxe à propos de la métonymie Nord-Sud: celle-ci semble très mal intégrer le rapport SudTravail. Ne dit-on pas (à tout le moins en pensée) que les gens du Sud ne travaillent guère? Ce propos ne fait pourtant pas très longtemps écran au rappel de l'invitation massive que les employeurs du Nord n'ont cessé (et ne cessent) d'adresser au Sud pour qu'il vienne s'embaucher à nos travaux les plus pénibles. Peut-on, dès lors, croire que c'est seulement en montant vers la forteresse nordique que l'esclave sudiste se met à trimer comme un bénédictin? Le Sud, qui soutient l'équilibre du Monde, devrait être au contraire associé à ce qui dans le "bas" veut plutôt dire "la base", à ce qui dans le "pauvre" renvoie plutôt à l'ascèse. Tout comme, lors de la grande implosion de la dominance romaine, le Sud désertique symbolisait - après avoir porté la Carthage concurrente désormais défunte - la cité de Dieu selon le Tunisien saint Augustin et les monachistes syriens, tout devrait nous convaincre que, pour adhérer avec conséquence à notre propre cosmologie, le Sud devrait être imaginé comme le socle même sur lequel la maîtrise est possible : à commencer par la maîtrise de soi. Et plus précisément, le Sud humain devrait être imaginé, dans la symbolique d'un univers appuyé sur lui, comme tout ce qui fait effort pour que l'équilibre universel s'assure, un peu comme la sagesse tantrique trouve sa racine corporelle à la base de l'épine dorsale. Pourquoi donc, cette idée d'un Sud lumineux - non de la lumière marchande du club touristique, mais de la clarté morale - semble-t-elle aujourd'hui si inaccessible, si ridicule même quand nous la rapportons à l'image banalisée des Méditerranéens jouisseurs ? Pourquoi donc le Sud continue-t-il, dans les idéaux inavouables de notre inconscient collectif, à rester synonyme de désordre, d'insouciance, de paresse, de violence, de débridage affectif, de 
pulsion terroriste et fanatique, d'indiscipline démographique, de faiblesse culturelle, etc?

D'autant que, si les régimes politiques y sont souvent autoritaires, les chiffres récents nous montrent que la démographie y est plus sage qu'on ne l'avait cru, que la sécurité des rues y est au moins aussi grande que dans nombre de villes du Nord, que les minorités ethniques et religieuses y ont souvent négocié de fort savants équilibres, que la culture s'y déploie dans une grande diversité d'oeuvres tout comme à travers un sens de l'humour qui, pour être mal connu vaut bien celui des Britanniques.

Pourquoi le maintien coûte que coûte d'images dépréciatives, lentement transformées en allégations d'agressivité à notre égard, d'un sud que la logique même de la symbolique d'un monde vertical devrait plutôt associer au salut racheté ?

Pour le dire autrement: pourquoi le Sud purificateur de Laurence d'Arabie, de Rimbaud, du père de Foucault ou de Cécile des Tentes ne s'est-il constitué que pour une élite ultra-réduite, alors qu'il devenait le lieu du marquage rageur des dérapages du Paris-Dakar, en attendant qu'y réponde, fatalement, le tir à balles réelles d'opposants aussitôt étiquetés de sournois?

Je ne vois qu'une seule réponse logique: parce que nous hésitons sur notre propre statut de Nordiques, et que les gens du Sud, vrais occupants de ces zones dures et sèches, nous rappellent certaines de nos plus intimes contradictions.

Occidentaux ou Nordistes? : le choix d'un éthos

Ce que nous voyons dans notre frère de l'Est quand il refuse de devenir maître de lui par le travail du capital, et nous montre une nouvelle mouture du couple boyard-serf, c'est un semblable égaré, un esclave qui rechigne devant l'ascèse de l'accumulation. Nous nous gardons de ses excès possibles à l'aide d'ICBM. En revanche, ce que suscite en nous le spectacle d'un Sud qui se lève, se modernise, dessale l'eau de mer à l'énergie nucléaire tout en tchadorisant ses femmes, c'est une insupportable contradiction : s'il travaille, lui aussi, alors que faire de notre propre ascèse patiemment distillée dans les siècles pour devenir vizirs à la place du vizir?

Si le Sud est ce qu'il doit être, du travail pur - et d'autant plus que son écologie est très fragile - alors quel sens prend notre millénaire d'effort productif si polluant, si destructeur, si coûteux, si terrible en termes de réduction de l'homme à l'individu, tout cela pour nous élever au rang de NORD ? Si les jambes se mettent à penser, où la tête va-t-elle aller se mettre?

Mais inversement, si nous empêchons le Sud de travailler, qui sommes-nous, nous qui avons tout misé sur la salvation de l'humanité par le travail, la Freud durch Arbeit d'un protestantisme placé depuis longtemps aux commandes d'une catholicité consentante? En effet, il est presque trivial de rappeler à quel point notre propre Maîtrise s'est construite depuis deux ou trois mille ans dans une lente perlaboration (Durcharbeit) pour éviter et dépasser le défi de combat à mort du Maître. Nous sommes en pratique d'anciens esclaves(12) ayant accédé au contrôle réciproque par l'acharnement à produire entre nous de vastes et complexes machines sociales et techniques. Nous ne pouvons oublier ce passé, pour aller jouer dans le sable. C'est la raison pour laquelle notre maitrise a un goût amer de labeur et de droit, un air d'interminable communication, qui n'évoque plus guère le style du grand guerrier, que nous avons détruit en nous à l'époque des Templiers. L'administration centrale de la monarchie devait en effet faire disparaître toute trace d'une possibilité de retour au pouvoir des 
purs combattants. Au nom des clercs, elle anticipait ainsi - et de ce fait donnait du sens - à ce qui deviendrait dans les démocraties modernes le principe d'une souveraineté du civil par rapport au militaire. Ce faisant, elle biffait pour longtemps le développement de la métonymie Nord-Sud, l'extinction des croisades coïncidant avec la promotion de la maîtrise interne, auto-centrée de type administratif, et le Krak des Chevaliers devenant du même coup symbole laissé en friche, d'un krach annoncé de la chevalerie, en attendant les exploits égyptiens de Napoléon. En reconstruisant en nous mêmes (dans nos territoires nordiques) l'opposition maître-sujet, nous nous prédestinions un jour à la projeter dans le miroitement Est-Ouest, dès le moment que, l'esclave étant devenu serf, et le serf bourgeois, le prolétaire ne pouvait que revendiquer rapidement son accès à la considération mutuelle, ce qu'il fit notamment au moyen du fulgurant raccourci historique de la scène soviétique, où le serf se proposa d'emblée comme apparatchik, sorte d'instituteur de l'histoire, venant se situer en vis-à-vis du chef d'entreprise occidental médusé.

Bien qu'étant passée à un cheveu de la destruction mutuelle assurée, la dialectique du miroir Ouest entrepreneur /Est bureaucrate s'est alors généralisée dans le Nord, de sorte qu'il a fini par apparaître évident à tout le monde qu'il y avait là une sorte de clivage fonctionnel de la civilisation des ex-esclaves, par lequel l'individualisme extrême du capitaliste ne pouvait survivre que grâce à une certaine dose de bureaucratie assurant des règles au marché, et qu'inversement, le bureaucrate ne pouvait survivre sans un principe de réalité extérieur à ses emprises sadiques: la liberté d'entreprendre. Dès lors que cette conscience se généralisait dans les profondeurs du "Peuple nordique", de Brest à Vladivostok en passant par San Francisco, on ne pouvait que dépasser la forme simple de cet équilibre: la menace nucléaire. L'effet de miroir Est-Ouest ne pouvait, tôt ou tard, que s'écrouler, laissant place à une holographisation généralisée des systèmes symboliques de relations: partout des entrepreneurs font désormais face à des bureaucrates, en une myriade de combats singuliers, de bras de force locaux, et l'individualisme le plus forcené se rencontre désormais bien davantage l'Est qu'à l'Ouest, à Gauche qu'à Droite.

C'est alors que la menace, en tant que condensation de l'identité a réclamé ses droits(13): car avec la pulvérisation des conflits dans l'hémisphère Nord, elle n'y retrouvait plus ce qui fait sa consistance : le miroitement unique, où l'on croit devoir sa propre existence unifiée à la haine-amour d'un Même situé en vis-à-vis.

21 Bien entendu, le Sud semblait disponible à ce jeu. Mais n'oublions pas que le nouveau maitre du Nord n'a plus rien à voir avec l'ancienne machine à défier et à tuer qu'étaient le guerrier odinique ou le chevalier teutonique. Il est hystérisé, coupé en deux volontés internalisées : celle du bureaucrate et celle de l'entrepreneur, tous les deux ex-esclaves. Pour cet être bifacial, creusé par sa propre histoire, traiter le Sud comme un simple réservoir d'esclaves ne peut donc que poser problème, et les aléas du colonialisme sont là pour le rappeler. De même que la réponse aux défis éventuellement posés par ceux des esclaves sudistes qui voudraient se porter désormais candidats à la maîtrise : les affronter en combat armé n'est pas sans faire question à celui qui y reconnaît ce qu'il a lui-même été dans le passé (avant l'industrialisation), et qu'il est toujours : quelqu'un qui préfère produire et vendre plutôt que quelqu'un qui extermine l'autre.

Voilà pourquoi la menace du Sud est menaçante: parce qu'elle force le Nordiste à oublier son propre passé d'esclave et de remontée vers la gloire par le rachat, pour redevenir un pur maître-guerrier, celui là même qu'Hegel avait rêvé. De la 
contradiction non assumée au délire de persécution. Il est de fait qu'en affrontant le candidat Saddam Hussein (dont on peut faire le curieux anagramme de : Sudd haï : menass), la coalition autour du maitre du Nord se prêtait à l'insistance de cette question : qui sommes-nous, des guerriers, des gens d'armes, ou bien des fabricants et des marchands?

En exemptant de service armé (contre impôt massif) les grandes seigneuries marchandes d'Allemagne et du Japon, l'Amérique a accentué encore l'analogie de la situation avec celles où les maîtres anciens exigeaient le ralliement de leurs vassaux à l'effort de guerre. Ce faisant, la question de la nature de la maîtrise moderne dans le monde s'est faite encore plus lancinante: sommes-nous des princes ou des hommes d'industrie?

Car si nous répondons par le deuxième terme, cela ne peut qu'ouvrir à la reconnaissance de la candidature du Sud au salut par le travail, même si cela s'accompagne d'une compétition accrue. En revanche, si nous répondons par le premier terme de l'alternative, nous entrons dans une zone de risques essentiels parce que notre identité moderne est menacée de délire.

Dans cette perspective, la question se déplace donc et devient face au Sud, restons-nous nous-mêmes, au risque de la concurrence, ou tuons nous celle-ci, en tuant, du même coup le principe de notre identité moderne?

L'inconscient de l'hésitation à reconnaître le Sud comme base de travail avoue alors son sens : car l'accepter, c'est du même coup trancher contre la tentation du retour à la maîtrise antique et féodale, et opter pour le maintien de l'éthique du capitalisme, celle des rédacteurs du Wall Street Journal dénonçant comme inhumaines et inconstitutionnelles les lois limitant l'immigration sud-américaine aux Etats-Unis.

Selon cette dernière, Autrui doit être reconnu comme sujet, fût-ce comme sujet à l'exploitation. Exploiter les gens du Sud, et pas seulement les ressources naturelles de leurs pays, implique alors l'émancipation hors de régimes dictatoriaux, car (bien qu'ignorée par les Chicago Boys), toute l'expérience du capitalisme le dit: les gens ne travaillent bien que dans un contexte social de relatives libertés politiques et sociales. Mais alors, nous devons reconnaître dans le Sud la présence d'Autrui dans les catégories de notre propre reconnaissance, et notamment celle de l'auto-salvation de l'esclave par le travail rémunérateur. Par exemple, nous devons nous accoutumer à banaliser l'image de Maghrébins membres du Rotary Club (Il n'y a pour l'instant qu'un représentant marocain pour tout le Maghreb !). Lors d'un récent passage à l'Heure de Vérité, Jacques Delors disait quelque chose que des décennies d'esprit de revanche - y compris dans les rangs du parti socialiste au pouvoir - avait rendu presque impossible à exprimer: à savoir qu il faut que nous appuyions résolument les démocrates maghrébins (laïcs compris) et l'islam véritable (tolérant et purement religieux) contre les intégrismes agressifs.

28 Même si cette idée (pourtant évidente depuis longtemps pour les amis des peuples méditérannéens) chemine désormais parmi les cercles dirigeants, on semble encore loin d'une réalisation à la mesure de l'importance de l'objectif. Malgré les nouvelles assez terrifiantes de la montée du FIS (Front Islamique du Salut) en Algérie et de sa capacité à mobiliser la jeunesse et les femmes, il semble que quelque chose résiste encore en nous à l'évocation d'un soutien sans réserve à ceux qui refusent ce populisme clérical. 
lextermination du resistant devient l'horizon inevitable, et sollicite d'abord son apparition comme pouvoir diabolisable. Le phantasme qui se déploie ici est un wishful thinking, une anticipation désirée d'une menace attendue, espérée pour permettre la riposte punitive : nous misons sur le fait que le développement des tensions qui suivrait d'éventuelles victoires politiques de l'intégrisme, ne pourrait mener qu'à une perspective de violences graves, et à une montée militariste soit en opposition, soit en appui la mouvance. II nous est aisé d'imaginer ensuite comment celle-ci s'articulerait à la série des conflits actuellement en émergence depuis la poudrière du Moyen-Orient jusqu'aux contrées soviétiques en cours de déstabilisations nationales et religieuses (Tadjikistan, Azerbaïdjan, etc.).

32 C'est alors que la réplique du Nord peut être glorieusement envisagée comme la dénégation du droit de l'Autre à l'égalité symbolique (celle de disposer de l'arme atomique, par exemple), et la population locale n'est imaginée comme foule fanatique que pour mieux devenir le support des coups symboliques à mort que nous désirons porter pour réduire l'adversaire à un statut inférieur, ou plutôt pour le faire rentrer dans son statut dominé. A travers les bombardements massifs, nous lançons un message écrit en lettres de flammes: "Tu n'existe pas comme notre semblable". Dès lors, le dictateur mis à mal, abaissé dans sa prétention à la maîtrise locale, doit se taire : mais on n'aidera pas pour autant le peuple à s'en émanciper, parce qu'il doit vivre la souffrance d'appartenir à la partie négative du monde.. Ainsi le peuple kurde ne sera pas réellement appuyé dans le sens émancipatif, mais utilisé pour faire diversion à l'occupation colonisante de la Palestine, et, accessoirement, pour faire apparaître le côté "humanitaire " du prince mondial.

"Tu n'existe pas, toi le Sud à travers le Musulman qui le représente.. Ce n'est que comme non-existant, non prétendant à la virilité que tu seras toléré". La menace non pas supprimée, mais déniée, la prestance rabattue par le mépris, distingue ainsi radicalement l'opposition Nord-Sud de celle entre Est et Ouest: Alors que la confrontation entre Orientaux et Occidentaux se concrétise par une dramatique course à l'identité (ne continue-t-on pas de reprocher le mimétisme aux Japonais ?), la menace du Sud n'est évoquée que pour être aussitôt tournée en dérision(14). Car il est entendu qu'elle ne doit une parcelle de réalité qu'à la duplicité des chimistes allemands ou des marchands d'armes français. Elle n'est qu'un lieu de réaffirmation indirecte et par de la pure prestance du Maître.

Mais aussitôt, derrière cette négation sans appel, une machinerie d'angoisse se met en place. Car le maitre actuel, comme je l'ai dit, garde la trace en lui de 1'ex-esclave, qui a si bien su se faire oublier par le travail. Que le nouveau dominateur du monde se 
souvienne de sa propre origine blessée, et il va également interdire à l'esclave nouveau de faire son salut par cette voie silencieuse du travail dont il connaitt tous les mystères. Ce que le guerrier ne réduira pas en cendre, la Banque mondiale viendra, en arrière, empêcher de croître par un endettement irrémissible.

Là encore, malaise dans le civilisé : sommes-nous réellement devenus ces êtres ambigus et contradictoires : guerrier la nuit, industriel le jour ? Sommes nous vraiment devenus ces monstres à deux têtes, incarnations géopolitiques du double-bind?

Les effets de folie guettent le maitre, par ricochet : car s'il ne laisse aucune place au travail de l'autre, il court le risque de détruire en lui-même la compétence du travail. Ne se prédestine-t-il pas à ne plus savoir lui-même travailler pour continuer sa propre rédemption historique?

Drôle de blague que celle où le raciste qui vient d'abattre un immigré entend la voix divine, au moment où il veut le dévaliser : "Le beur, oui ! mais pas l'argent du beur !" Car cet argent, il est clair qu'il l'a gagné par un travail que le Français ne voudrait plus faire, mais qui relève néanmoins d'un respect depuis longtemps intériorisé pour le... labeur. Le jeu de mot(15) nous met donc ici directement au coeur du problème : qu'en attaquant l'esclave, celui qui ne prétend à aucune maîtrise mais à sa seule dignité, on attaque le travail, cette origine même de notre propre identité.

La menace du Sud apparaît donc dans sa vraie place : nous-mêmes et dans sa vérité, nous distinguons notre folie.. Car, sitôt que nous sommes piégés dans cette soi-disant évidence d une montée agressive du Sud vers le Nord, du travail (nié comme tel) vers la c'est très logiquement que maitrise paresseuse (niée comme telle), vient l'hallucination de la persécution provoquée par la privation de travail : au coeur même de la capitale fédérale américaine, la figure du Sud dévalise les supermarchés, au coeur des banlieues domestiques françaises, l'enfant du travailleur maghrébin fait émeute. Le Sud envahit du moment où il se présente en consommateur exsangue aux portes de l'abondance qu'un flux ininterrompu d'immigrés a permis de produire.

La vacillation entre les deux conceptions de la maitrise (antique et moderne) à laquelle nous confronte le Sud s'explique aussi par la maturation du travail, et son incidence, par le biais des hautes technologies, sur l'expulsion des travailleurs hors des processus productifs. Nous sommes ainsi d'autant plus disponibles à la tentation du souvenir très ancien du tyran collectif sans souci de discipline productive, que nos propres machines nous ont peu à peu évincés nous-mêmes de l'activité intéressante ou utile. Car le monde antique nous rappelle l'étroite dialectique de l'oisiveté et de la maîtrise, le maître étant avant tout celui qui exclut, et non pas celui qui donne du travail.

Au-delà de la menace Nord-Sud : l'accident de l'Occident

40 La menace réelle est donc l'incertitude d'un monde d'où des solutions déjà éprouvées comme e travail civilisateur - ont été conduites à leur ultima ratio, paradoxale comme il se doit. Mais nous ne voyons toujours pas à quoi pourrait ressembler un monde sans maitres, qu'ils soient guerriers ou industriels. Nous ne croyons plus guère à l'alternative des clercs, prêtres ou universitaires, qui ne sont généralement que des acolytes, des accompagnateurs des grands pouvoirs, à moins qu'ils ne fassent euxmêmes des maîtres éclairés, parFois jusqu'à l'illuminisme : sombre clarté qui tombe des stalines !. Bref, les triades à la Dumézil nous semblent avoir épuisé leur capacité d'autorégulation. C'est là qu'est le contenu le plus réel de la menace qu'illustre la brèche Nord-Sud : une faille dans notre propre culture. 
41 Le perçoit-on assez ? Voit-on assez que le fait de pointer le danger d'un candidat-maître au Sud, valeureux mais détestable émule de nos fantasmes de domination, disséminateur démoniaque de virus manipulés, copieur de bombes à souffle plus ou moins court, cela nous oriente vers un abandon de nos laborieuses éthiques, et une retrouvaille avec la passion guerrière, dont A. Hirschman a montré combien le capitalisme a mis de soin à se défaire par son transfert dans la passion utilitaire ?(16).

Et comme le maître mondial peut se faire incontestable par la sophistication de son armement, ne voit-on pas que c'est à l'apologie de la royale fénéantise, négation même de tout notre devenir historique, que conduit l'ennui du Maître, une fois bridée la révolte servile?

A ce point qu'on peut se demander si, lorsque nous imitons notre Ami américain dans l'augmentation de nos déficits commerciaux, ce qui nous motive - loin de relever d'une simple fatigue industrielle n'est pas le très ancien idéal selon lequel un vrai maître est celui qui se fait porter, soutenir -passif et halluciné- par le reste du monde?

\section{RÉSUMÉS}

Si l'Est peut être considéré comme un double de l'Ouest et leur rivalité comme un jeu de miroir, le conflit avec le " Sud " pose le problème de l'identité même du " Nord " et renvoie ce dernier à ses contradictions car le " Sud ", en tant que candidat au tra vail, reflète le passé du " Nord ". Ainsi, la menace du " Sud " piège-t-elle le " Nord " en l'isolant dans sa propre angoisse.

If the East can be considered a duplicate of the West and their rivalry a mirror game, the conflict with the " South " questions the " North's " self-identity and sends it back to its internal contradictions. For the " South ", as a candidate for work, is reflecting the " North's " past. Thus, the " Southern threat " traps the " North" and confines it in its own distresses.

\section{INDEX}

Mots-clés : menace, discours sécuritaire

Index géographique : Pays du Sud (Tiers Monde), Relations Nord/ Sud

\section{AUTEUR}

\section{DENIS DUCLOS}

Chercheur au CNRS, Directeur du groupe de recherches : sociétés et risques scientifiques et techniques 